Volume 24 (2018) 31-49

DOI: $10.24330 /$ ieja.440164

\title{
ABSORBING COMULTIPLICATION MODULES OVER A PULLBACK RING
}

\author{
S. Ebrahimi Atani, S. Dolati Pish Hesari, M. Khoramdel and \\ M. Sedghi Shanbeh Bazari \\ Received: 28 September 2017; Revised: 18 December 2017; Accepted: 21 December 2017 \\ Communicated by Alberto Facchini \\ Dedicated to the memory of Professor John Clark

\begin{abstract}
The purpose of this paper is to introduce the concept of 2-absorbing comultiplication modules, which form a subclass of the class of pure-injective modules over pullback rings. A full description of all indecomposable 2absorbing comultiplication modules with finite-dimensional top over the pullback of two discrete valuation domains with the same residue field is given.
\end{abstract}

Mathematics Subject Classification (2010): 03C45, 03C05, 16D70

Keywords: Pullback, separated, non-separated, 2-absorbing comultiplication module, pure-injective module

\section{Introduction}

Over most rings it is impossible to classify all modules: even algebras of tame representation type typically are "wild" when their infinitely generated representations are considered. In particular, one is interested in the classification of certain "significant" modules rather than in arbitrary modules. The pure-injective modules seem to form a class of modules that appear naturally and where there is hope of some kind of classification. Pure-injective modules play a central role in the model theory of modules: for example classification of the complete theories of $R$-modules reduce to classifying the (complete theories of) pure-injectives. Also, for some rings the "small" (finite-dimensional, finitely generated, ...) modules are classified and in many cases this classification can be extended to give a classification of the (indecomposable) pure-injective modules. Indeed, there is sometimes a strong connection between infinitely generated pure-injective modules and families of finitely generated modules (see [31], [32], [33], [34] and [40]). The reader is referred to [3], [35, Chapters 1 and 14] and [36] for a detailed discussion of classification problems, their representation types (finite, tame, or wild), and useful computational reduction procedures. 
In this paper all rings are commutative with identity and all modules unitary. We are going to study pullbacks of discrete valuation rings. Let $v_{1}: R_{1} \rightarrow \bar{R}$ and $v_{2}: R_{2} \rightarrow \bar{R}$ be homomorphisms of two discrete valuation domains $R_{i}$ onto a common field $\bar{R}$. Denote the pullback $R=\left\{\left(r_{1}, r_{2}\right) \in R_{1} \oplus R_{2}: v_{1}\left(r_{1}\right)=v_{2}\left(r_{2}\right)\right\}$ by $\left(R_{1} \stackrel{v_{1}}{\longrightarrow} \bar{R} \stackrel{v_{2}}{\longleftarrow} R_{2}\right)$, where $\bar{R}=R_{1} / J\left(R_{1}\right)=R_{2} / J\left(R_{2}\right)$. Then $R$ is a ring under coordinate-wise multiplication. Denote the kernel of $v_{i}, i=1,2$, by $P_{i}$. Then $\operatorname{Ker}(R \rightarrow \bar{R})=P=P_{1} \times P_{2}, R / P \cong \bar{R} \cong R_{1} / P_{1} \cong R_{2} / P_{2}$, and $P_{1} P_{2}=P_{2} P_{1}=0$ (so $R$ is not a domain). Furthermore, for $i \neq j, 0 \rightarrow P_{i} \rightarrow R \rightarrow R_{j} \rightarrow 0$ is an exact sequence of $R$-modules (see [24]). A typical example of pullback of discrete valuation domain is the infinite-dimensional $k$-algebra $k[x, y: x y=0]_{(x, y)}$ where $k$ is a field (it is the pullback $\left(k[x]_{(x)} \rightarrow k \leftarrow k[y]_{(y)}\right)$ of two discrete valuation domains $k[x]_{(x)}, k[y]_{(y)}$ (see $[2$, Section 6$]$ ). Let $R$ be a pullback of two discrete valuation domains with common residue field $K$. As in [24] define the associated graded ring $G(R)$ to be the additive group $\oplus_{i} P^{i} / P^{i+1}$ equipped with a ring structure by defining the multiplication as in [24]. Similarly, the associated graded module of an $R$-module $M$ is $P^{i} M / P^{i+1} M$, equipped with a $G(R)$-module structure by defining the scalar multiplication as in [24]. Arnold and Laubenbacher ([2, Section 6]) showed that $G(R)$ is the $k$-algebra $k[x, y: x y=0]_{(x, y)}$. The $R$-modules of deleted and block cycle types correspond exactly to the $G(R)$-modules of string and band types (see [8]). Modules over pullback rings have been studied by several authors (see for instance, [2], [7], [10], [12], [14], [15], [16], [17], [19], [20], [21], [23], [24], [25], [26], [29], [38]). Notably, there is the important work of Levy [25], resulting in the classification of all finitely generated indecomposable modules over Dedekind-like rings. Common to all these classification is the reduction to a "matrix problem" over a division ring, see [9] and [35, Section 17.9] for a background of matrix problems and their applications. It is proved that the pullback of two commutative local rings has Morita duality if and only if the two rings have Morita duality ([18]).

The classification of subclass of pure-injective modules over the pullback of two discrete valuation rings over a common factor field are very important and a difficult problem. One point of this paper is that to introduce a subclass of pure-injective modules over such rings. Indeed, this article includes the classification of those indecomposable 2-absorbing comultiplication modules over $k[x, y: x y=0]_{(x, y)}$ where $k$ is a field, which have finite-dimensional top.

In the present paper, we introduce a new class of $R$-modules, called 2-absorbing comultiplication modules, and we study it in details from the classification problem point of view. We are mainly interested in case either $R$ is a discrete valuation domain or $R$ is a pullback of two discrete valuation domains. First, we give a complete 
description of the 2-absorbing comultiplication modules over a discrete valuation domain. Let $R$ be a pullback of two discrete valuation domains over a common factor field. The main purpose of this paper is to give a complete description of the indecomposable 2-absorbing comultiplication $R$-modules with finite-dimensional top over $R / \operatorname{rad}(R)$ (for any module $M$ we define its top as $M / \operatorname{Rad}(R) M$ ). The classification is divided into two stages: the description of all indecomposable separated 2-absorbing comultiplication $R$-modules and then, using this list of separated 2-absorbing comultiplication modules we show that non-separated indecomposable 2 -absorbing comultiplication $R$-modules with finite-dimensional top are factor modules of finite direct sums of separated indecomposable 2-absorbing comultiplication $R$-modules. Then we use the classification of separated indecomposable 2-absorbing comultiplication modules from Section 3, together with results of Levy [25,26] on the possibilities of amalgamating finitely generated separated modules, to classify the non-separated indecomposable 2-absorbing comultiplication modules with finitedimensional top (see Theorem 4.7). We will see that the non-separated modules may be represented by certain amalgamation chains of separated indecomposable 2-absorbing comultiplication modules (where infinite length 2-absorbing comultiplication modules can occur only at the ends) and where adjacency corresponds to amalgamation in the socles of these separated 2-absorbing comultiplication modules.

The concept of 2-absorbing ideal, which is a generalization of that of prime ideal, was introduced and studied by Badawi in [4]. Various generalizations of prime ideals are also studied in [5] and [6]. Recall that a proper ideal $I$ of a ring $R$ is called a 2-absorbing ideal of $R$ if whenever $a, b, c \in R$ and $a b c \in I$, then $a b \in I$ or $a c \in I$ or $b c \in I$. Recently (see [30], [39]), the concept of 2-absorbing ideal is extended to the context of 2-absorbing submodule which is a generalization of prime submodule. Recall from [30] that a proper $R$-submodule $N$ of a module $M$ is said to be a 2-absorbing submodule of $M$ if whenever $a, b \in R, m \in M$ and $a b m \in N$, then $a m \in N$ or $b m \in N$ or $a b \in\left(N:_{R} M\right)$.

For the sake of completeness, we state some definitions and notations used throughout. Let $R$ be the pullback ring as mentioned in the beginning of introduction. An $R$-module $S$ is defined to be separated if there exist $R_{i}$-modules $S_{i}$, $i=1,2$, such that $S$ is a submodule of $S_{1} \oplus S_{2}$ (the latter is made into an $R$-module by setting $\left.\left(r_{1}, r_{2}\right)\left(s_{1}, s_{2}\right)=\left(r_{1} s_{1}, r_{2} s_{2}\right)\right)$. Equivalently, $S$ is separated if it is a pullback of an $R_{1}$-module and an $R_{2}$-module and then, using the same notation for pullbacks of modules as for rings, $S=\left(S / P_{2} S \rightarrow S / P S \leftarrow S / P_{1} S\right)$ [24, Corollary 3.3] and $S \subseteq\left(S / P_{2} S\right) \oplus\left(S / P_{1} S\right)$. Also $S$ is separated if and only if $P_{1} S \cap P_{2} S=0$ 
[24, Lemma 2.9]. Let $M$ be an $R$-module. A separated representation of $M$ is a pair $(S, \varphi)$ where

(i) $S$ is a separated $R$-module;

(ii) $\varphi$ is an $R$-homomorphism of $S$ onto $M$;

(iii) for every pair $\left(S^{\prime}, \varphi^{\prime}\right)$ satisfying (i) and (ii), and for every $R$-homomorphism $\alpha$ of $S$ in $S^{\prime}$ such that $\varphi^{\prime} \alpha=\varphi, \alpha$ is $1-1$. The module $K=\operatorname{Ker}(\varphi)$ is then an $\bar{R}$-module, since $\bar{R}=R / P$ and $P K=0$ [24, Proposition 2.3]. An exact sequence $0 \rightarrow K \rightarrow S \rightarrow M \rightarrow 0$ of $R$-modules with $S$ separated and $K$ an $\bar{R}$-module is a separated representation of $M$ if and only if $P_{i} S \cap K=0$ for each $i$ and $K \subseteq P S$ [24, Proposition 2.3]. Every module $M$ has a separated representation, which is unique up to isomorphism [24, Theorem 2.8].

Definition 1.1. (a) If $R$ is a ring and $N$ is a submodule of an $R$-module $M$, the ideal $\{r \in R: r M \subseteq N\}$ is denoted by $(N: M)$. Then $(0: M)$ is the annihilator of $M$. A proper submodule $N$ of a module $M$ over a $\operatorname{ring} R$ is said to be prime submodule if whenever $r m \in N$, for some $r \in R, m \in M$, then $m \in N$ or $r \in(N: M)$, so $(N: M)=P$ is a prime ideal of $R$, and $N$ is said to be $P$-prime submodule. The set of all prime submodules in an $R$-module $M$ is denoted by $\operatorname{Spec}(M)[27,28]$.

(b) An $R$-module $M$ is a comultiplication module provided for each submodule $N$ of $M$ there exists an ideal $I$ of $R$ such that $N$ is the set of elements $m$ in $M$ such that $\operatorname{Im}=0$. In this case we can take $N=\left(0:_{M} \operatorname{ann}(N)\right)[1]$.

(c) An $R$-module $M$ is defined to be a weak comultiplication module if $\operatorname{Spec}(M)=$ $\emptyset$ or for every prime submodule $N$ of $M, N=\left(0:_{M} I\right)$, for some ideal $I$ of $R$ [15].

(d) A proper submodule $N$ of a module $M$ is said to be 2-absorbing submodule if whenever $a, b \in R, m \in M$ and $a b m \in N$, then $a m \in N$ or $b m \in N$ or $a b \in\left(N:_{R}\right.$ $M)[30,39]$. The set of all 2-absorbing submodules in an $R$-module $M$ is denoted by $\operatorname{abSpec}(M)$.

(e) A submodule $N$ of an $R$-module $M$ is called a pure submodule if any finite system of equations over $N$ which is solvable in $M$ is also solvable in $N$. A submodule $N$ of an $R$-module $M$ is called relatively divisible (or an $R D$-submodule) in $M$ if $r N=N \cap r M$ for all $r \in R$ (see [31], [37]).

(g) A module $M$ is pure-injective if it has the injective property relative to all pure exact sequences (see [31], [37]).

Remark 1.2. (a) Let $R$ be a Dedekind domain, $M$ an $R$-module and $N$ a submodule of $M$. Then $N$ is pure in $M$ if and only if $I N=N \cap I M$ for each ideal $I$ of $R$. Moreover, $N$ is pure in $M$ if and only if $N$ is an RD-submodule of $M$ [31], [37]. 
(b) Let $N$ be an R-submodule of $M$. It is clear that $N$ is an $R D$-submodule of $M$ if and only if for all $m \in M$ and $r \in R, r m \in N$ implies that $r m=r n$ for some $n \in N$. Furthermore, if $M$ is torsion-free, then $N$ is an $R D$-submodule if and only if for all $m \in M$ and for all non-zero $r \in R, r m \in N$ implies that $m \in N$. In this case, $N$ is an RD-submodule if and only if $N$ is a prime submodule.

\section{2-Absorbing comultiplication modules}

In this section, we give a complete description of the 2-absorbing comultiplication modules over a discrete valuation domain. We begin with the key definition of this paper.

Definition 2.1. Let $R$ be a commutative ring. An $R$-module $M$ is defined to be a 2-absorbing comultiplication module if $\operatorname{abSpec}(M)=\emptyset$ or for every 2-absorbing submodule $N$ of $M, N=\left(0:_{M} I\right)$, for some ideal $I$ of $R$.

One can easily show that if $M$ is a 2-absorbing comultiplication module, then $N=\left(0:_{M} \operatorname{ann}(N)\right)$ for every 2-absorbing submodule $N$ of $M$. It is easy to see that the class of 2-absorbing comultiplication modules contains the class of weak comultiplication modules (resp. comultiplication modules) defined in [15] (resp. [14]). We need the following lemma proved in [39, Lemma 2.4] and [30, Lemma 2.1, Lemma 2.2, and Theorem 2.3], respectively.

Lemma 2.2. $\quad$ (i) Let $K \subseteq N$ be submodules of an $R$-module $M$. Then $N$ is a 2 -absorbing submodule of $M$ if and only if $N / K$ is a 2-absorbing submodule of $M / K$.

(ii) Let $I$ be an ideal of $R$ and $N$ be a 2-absorbing submodule of $M$. If $a \in R$, $m \in M$ and $I a m \subseteq N$, then $a m \in N$ or $\operatorname{Im} \subseteq N$ or $I a \subseteq(N: M)$.

(iii) Let $I, J$ be ideals of $R$ and $N$ be a 2-absorbing submodule of $M$. If $m \in M$ and $I J m \subseteq N$, then $I m \in N$ or $J m \subseteq N$ or $I J \subseteq(N: M)$.

(iv) Let $N$ be a proper submodule of $M$. Then $N$ is a 2-absorbing submodule of $M$ if and only if $I J K \subseteq N$ for some ideals $I, J$ of $R$ and a submodule $K$ of $M$ implies that $I K \subseteq N$ or $J K \subseteq N$ or $I J \subseteq(N: M)$.

Lemma 2.3. $\quad$ (a) Let $M$ be an R-module, $N$ an $R$-submodule of $M$ and $I$ an ideal of $R$ such that $I \subseteq \operatorname{ann}(M)$. Then $M$ is a 2-absorbing comultiplication $R$-module if and only if $M$ is 2-absorbing comultiplication as an $R / I$-module.

(b) Let $R$ and $R^{\prime}$ be any commutative rings, $g: R \rightarrow R^{\prime}$ a surjective homomorphism and $M$ an $R^{\prime}$-module. If $M$ is a 2-absorbing comultiplication $R^{\prime}$-module, then $M$ is a 2-absorbing comultiplication $R$-module. 
Proof. (a) It is easy to see that $N$ is a 2-absorbing $R$-submodule of $M$ if and only if $N$ is 2-absorbing submodule of $M$ as an $R / I$-module. Now the assertion follows the fact that $\left(0:_{M} J\right)=\left(0:_{M}(I+J) / I\right)$ for every ideal $J$ of $R$.

(b) Clearly, if $N$ is a 2-absorbing $R$-submodule of $M$, then it is a 2-absorbing $R^{\prime}$-submodule of $M$. Assume that $M$ is a 2-absorbing comultiplication $R^{\prime}$-module and let $N$ be a 2-absorbing $R$-submodule of $M$. Then $N=\left(0:_{M} J\right)$, where $J=\left(0::_{R^{\prime}} N\right)$; so $I=g^{-1}(J)$ is an ideal of $R$ with $g(I)=J$. It is enough to show that $\left(0:_{M} J\right)=\left(0:_{M} I\right)$. Let $m \in\left(0:_{M} J\right)$. If $r \in I$, then $g(r) \in J$, so $g(r) m=0$. Thus $r m=0$ for every $r \in I$; hence $m \in\left(0:_{M} I\right)$. For the reverse inclusion, assume that $x \in\left(0:_{M} I\right)$ and $s \in J$. Then $s=g(a)$ for some $a \in I$. It follows that $s x=g(a) x=a x=0$ for every $s \in J$; hence $x \in\left(0:_{M} J\right)$, and we have equality.

Proposition 2.4. Assume that $M$ is a 2-absorbing comultiplication module over a commutative ring $R$ and let $N$ be a non-zero pure submodule of $M$. Then the following hold:

(i) $M / N$ is a 2-absorbing comultiplication $R$-module.

(ii) Every direct summand of $M$ is a 2-absorbing comultiplication submodule.

Proof. (i) Let $K / N$ be a 2-absorbing submodule of $M / N$. Then by Lemma 2.2(i), $K$ is a 2-absorbing submodule of $M$, so $K=\left(0:_{M} I\right)$ for some ideal $I$ of $R$. We show that $K / N=\left(0:_{M / N} I\right)$. Let $x+N \in K / N$. Then $I x=0$ gives $I(x+N)=0$; so $x+N \in\left(0:_{M / N} I\right)$. For the reverse inclusion, assume that $y+N \in\left(0:_{M / N} I\right)$. Then $I y \subseteq N \cap I M=I N \subseteq I K=0$; hence $y \in K$, and we have equality.

(ii) follows from (i).

Remark 2.5. Let $R$ be a discrete valuation domain with unique maximal ideal $P=R p$.

(a) Since $P$ is a 2-absorbing submodule of $R$-module $R$ with $\left(0:_{R} \operatorname{ann}(P)\right)=$ $R \neq P$, we get that $R$ is not a 2-absorbing comultiplication $R$-module.

(b) $B y[17, \operatorname{Proposition~2.5],~} \operatorname{abSpec}(E(R / P))=\emptyset$ and $\operatorname{abSpec}(Q(R))=\{(0)\}$ (so $\{0\}=\left(0:_{Q(R)} R\right)$ ), where $E(R / P)$, the injective hull of $R / P$ and $Q(R)$, the field of fractions of $R$. Thus they are 2-absorbing comultiplication $R$ modules.

(c) Each $R / P^{n}(n \geq 1)$ is a 2-absorbing comultiplication module since it is a comultiplication module (see [14]). 
Theorem 2.6. Let $R$ be a discrete valuation domain with a unique maximal ideal $P=R p$. Then the indecomposable 2-absorbing comultiplication modules over $R$, up to isomorphism, are the following:

(i) $R / P^{n}, n \geq 1$, the indecomposable torsion modules;

(ii) $E(R / P)$, the injective hull of $R / P$;

(iii) $Q(R)$, the field of fractions of $R$.

Proof. By [10, Proposition 1.3], these modules are indecomposable. They are 2-absorbing comultiplication by Remark 2.5. It remains to be shown that there are no more indecomposable 2-absorbing comultiplication modules. Let $M$ be an indecomposable 2-absorbing comultiplication and choose a non-zero element $a \in M$. Consider the annihilator, $\operatorname{ann}_{R}(a)=\{r \in R: r a=0\}$, and the height $h(a)=$ $\sup \left\{n: a \in P^{n} M\right\}$ (so $h(a)$ is a non-negative integer or $\infty$ ). If $\operatorname{ann}_{R}(a)=P^{m+1}$, then $\operatorname{ann}_{R}\left(a p^{m}\right)=P$. So, replacing $a$ if necessary, it may be supposed that $\operatorname{ann}_{R}(a)$ is 0 or $P$. Now we consider the various possibilities for $h(a)$ and $\operatorname{ann}_{R}(a)$.

Case 1: If $\operatorname{abSpec}(M)=\emptyset$, then $\operatorname{Spec}(M) \subseteq \operatorname{abSpec}(M)$ gives $M$ is a torsion divisible $R$-module with $P M=M$ and $M$ is not finitely generated by [27, Lemma 1.3, Proposition 1.4]. We may assume that $(0: a)=P$. By an argument like that in [11, Proposition 2.7 Case 2], $M \cong E(R / P)$. So we may assume that $\operatorname{abSpec}(M) \neq \emptyset$.

Case 2: If $h(a)=n$, then $\operatorname{ann}_{R}(a)=P$. Assume to the contrary, $\operatorname{ann}_{R}(a)=0$. Say $a=p^{n} b$. Then $r b=0$ implies $r a=0$ and so $r=0$. Thus $R b \cong R$. We also have that $R b$ is pure in $M$ (see [13, Theorem 2.12 Case 1]). As $M$ is a torsion-free $R$-module by [22, Theorem 10], we must have $R b$ is a prime submodule of $M$ (see Remark 1.2 (b)) (so 2-absorbing submodule); hence $R \cong R b=\left(\begin{array}{ll}0:_{M} & 0\end{array}\right)=M$, which is a contradiction by Remark 2.5 (a). So we may assume that $h(a)=n$, $(0: a)=P$. Say $a=p^{n} b$. Then we have $R b \cong R / P^{n+1}$. Furthermore, $R b$ is pure in $M$. Hence, since $R b$ is a pure submodule of bonded order of $M$, we deduce that $R b$ is a direct summand of $M$ by [22, Theorem 5]; hence $M=R b \cong R / P^{n+1}$.

Case 3: $h(a)=\infty, \operatorname{ann}_{R}(a)=P$. By an argument like that [11, Theorem 2.12, Case 4], we get that $M \cong E(R / P)$. Hence $\operatorname{abSpec}(M)=\emptyset$ by Remark 2.5 that is a contradiction.

Case 4: $h(a)=\infty, \operatorname{ann}_{R}(a)=0$. By an argument like that [13, Theorem 2.12, Case 3], we obtain $M \cong Q(R)$.

Theorem 2.7. Let $M$ be a 2-absorbing comultiplication module over a discrete valuation domain with a unique maximal ideal $P=R p$. Then $M$ is of the form $M=N \oplus K$, where $N$ is a direct sum of copies of $R / P^{n}(n \geq 1)$ and $K$ is 
a direct sum of copies of $E(R / P)$ and $Q(R)$. In particular, every 2-absorbing comultiplication $R$-module is pure-injective.

Proof. Let $T$ denote an indecomposable summand of $M$. Then $T$ is an indecomposable 2-absorbing comultiplication module by Proposition 2.4(ii). Now the assertion follows from Theorem 2.6. The "in particular" statement follows from [10, Proposition 1.3].

\section{The separated absorbing comultiplication modules}

In this section we determine the indecomposable absorbing comultiplication separated $R$-modules where

$$
R=\left(R_{1} \stackrel{v_{1}}{\longrightarrow} \bar{R} \stackrel{v_{2}}{\longleftarrow} R_{2}\right)
$$

is the pullback of two discrete valuation domains $R_{1}, R_{2}$ with maximal ideals $P_{1}, P_{2}$ generated respectively by $p_{1}, p_{2}, P$ denotes $P_{1} \oplus P_{2}$ and $R_{1} / P_{1} \cong R_{2} / P_{2} \cong R / P \cong \bar{R}$ is a field (we do not need the a priori assumption of finite-dimensional top for this classification). Then $R$ is a commutative Noetherian local ring with unique maximal ideal $P$. The other prime ideals of $R$ are easily seen to be $P_{1}$ (that is $\left.P_{1} \oplus 0\right)$ and $P_{2}$ (that is $0 \oplus P_{2}$ ). Let $a=(r, s) \in R$ with $r \neq 0$ and $s \neq 0$. Then we can write $a=\left(p_{1}^{n}, p_{2}^{m}\right)$ for some positive integers $m, n$, so $\operatorname{ann}(a)=0$; hence $R a \cong R$. If $a=\left(0, p_{2}^{m}\right)$ for some positive integer $m$, then $\operatorname{ann}(a)=P_{1} \oplus 0$, and so $R\left(0, p_{2}^{m}\right) \cong R /\left(P_{1} \oplus 0\right) \cong R_{2}$. Similarly, $R\left(p_{1}^{n}, 0\right) \cong R /\left(0 \oplus P_{2}\right) \cong R_{1}$. The other ideals $I$ of $R$ are of the form $I=P_{1}^{n} \oplus P_{2}^{m}=\left(P_{1}^{n}, P_{2}^{m}\right)=\left(<p_{1}^{n}>,<p_{2}^{m}>\right)$ for some positive integers $m, n$ since $I \subseteq P=P_{1} \oplus P_{2}=\left(P_{1}, P_{2}\right)=\left(<p_{1}>,<p_{2}>\right)$ and $p_{1} p_{2}=0=p_{2} p_{1}$ (see [10, p. 4054]). We need the following lemma proved in [17, Proposition 3.1].

Lemma 3.1. Let $S=\left(S / P_{2} S=S_{1} \stackrel{f_{1}}{\longrightarrow} \bar{S}=S / P S \stackrel{f_{2}}{\longleftarrow} S_{2}=S / P_{1} S\right)$ be any separated module over the pullback ring as in (1).

(i) If $T$ is a 2-absorbing submodule of $S$, then $T_{1}$ is a 2-absorbing submodule of $S_{1}$ and $T_{2}$ is a 2-absorbing submodule of $S_{2}$.

(ii) $\operatorname{abSpec}(S)=\emptyset$ if and only if $\operatorname{abSpec}\left(S_{i}\right)=\emptyset$ for $i=1,2$.

Remark 3.2. Let $R$ be the pullback ring as in (1), and let $T$ be an $R$-submodule of a separated module $S=\left(S_{1} \stackrel{f_{1}}{\longrightarrow} \bar{S} \stackrel{f_{2}}{\longleftarrow} S_{2}\right)$, with projection maps $\pi_{i}: S \rightarrow S_{i}$. Set $T_{1}=\left\{t_{1} \in S_{1}:\left(t_{1}, t_{2}\right) \in T\right.$ for some $\left.t_{2} \in S_{2}\right\}$ and $T_{2}=\left\{t_{2} \in S_{2}:\left(t_{1}, t_{2}\right) \in\right.$ $T$ for some $\left.t_{1} \in S_{1}\right\}$. Then for each $i, i=1,2, T_{i}$ is an $R_{i}$-submodule of $S_{i}$ and $T \leq$ $T_{1} \oplus T_{2}$. Moreover, we can define a mapping $\pi_{1}^{\prime}=\pi_{1} \mid T: T \rightarrow T_{1}$ by sending $\left(t_{1}, t_{2}\right)$ to $t_{1}$. Hence $T_{1} \cong T /\left(0 \oplus \operatorname{Ker}\left(f_{2}\right) \cap T\right) \cong T /\left(T \cap P_{2} S\right) \cong\left(T+P_{2} S\right) / P_{2} S \subseteq S / P_{2} S$. 
So we may assume that $T_{1}$ is a submodule of $S_{1}$. Similarly, we may assume that $T_{2}$ is a submodule of $S_{2}$ (note that $\operatorname{Ker}\left(f_{1}\right)=P_{1} S_{1}$ and $\operatorname{Ker}\left(f_{2}\right)=P_{2} S_{2}$ ).

Proposition 3.3. Let $R$ be the pullback ring as in (1), and let $S=\left(S_{1} \rightarrow \bar{S} \leftarrow S_{2}\right)$ be any separated $R$-module. Then the following hold:

(i) If $L_{1}$ is a non-zero 2-absorbing submodule of $S_{1}$, then there exists a separated submodule $T$ of $S$ such that $T+\left(0 \oplus P_{2}\right) S$ is a 2-absorbing submodule of $S$.

(ii) If $L_{2}$ is a non-zero 2-absorbing submodule of $S_{2}$, then there exists a separated submodule $T^{\prime}$ of $S$ such that $T^{\prime}+\left(P_{1} \oplus 0\right) S$ is a 2-absorbing submodule of $S$.

Proof. (i) If $L_{1}$ is a non-zero 2-absorbing submodule of $S_{1}$, then there exists a separated submodule $T=\left(T_{1} \rightarrow \bar{T} \leftarrow T_{2}\right)$ of $S$, where $T_{1}=L_{1}$. By Remark 3.2, $T_{1} \cong\left(T+\left(0 \oplus P_{2}\right) S\right) /\left(0 \oplus P_{2}\right) S \subseteq S /\left(0 \oplus P_{2}\right) S$. Thus $\left(T+\left(0 \oplus P_{2}\right) S\right) /\left(0 \oplus P_{2}\right) S$ is a 2-absorbing $R$-submodule of $S /\left(0 \oplus P_{2}\right) S$. Hence $T+\left(0 \oplus P_{2}\right) S$ is a 2-absorbing $R$-submodule of $S$ by Lemma 2.2(i). The proof of (ii) is similar.

Theorem 3.4. Let $S=\left(S_{1} \rightarrow \bar{S} \leftarrow S_{2}\right)$ be any separated module over the pullback ring as (1). Then $S$ is a 2-absorbing comultiplication $R$-module if and only if each $S_{i}$ is a 2-absorbing comultiplication $R_{i}$-module, $i=1,2$.

Proof. By Lemma 3.1(ii), we may assume that $\operatorname{abSpec}(S) \neq \emptyset$. Suppose that $S$ is a 2-absorbing comultiplication $R$-module and let $L$ be a nonzero 2-absorbing submodule of $S_{1}$. By Proposition 3.3, there exists a submodule $T=\left(T_{1} \rightarrow \bar{T} \leftarrow T_{2}\right)$ of $S$ such that $L=T_{1}$ and $T^{\prime}=T+\left(0 \oplus P_{2}\right) S$ is a 2-absorbing submodule of $S$. Clearly, $\operatorname{ann}\left(T^{\prime}\right)=\operatorname{ann}(T) \cap \operatorname{ann}\left(\left(0 \oplus P_{2}\right) S\right)=0$ or $P_{1}^{n} \oplus 0$ for some positive integer $n$. Since $S=(0: S 0), S$ is a 2-absorbing comultiplication module gives $T^{\prime}=\left(0:_{S} P_{1}^{n} \oplus 0\right)$. It suffices to show that $L=T_{1}=\left(0::_{S_{1}} p_{1}^{n}\right)$. Let $t \in T_{1}$. There exists $t_{2} \in T_{2}$ such that $\left(t_{1}, t_{2}\right) \in T \subseteq T^{\prime}$; so $\left(P_{1}^{n} \oplus 0\right)\left(t_{1}, t_{2}\right)=0$. It then follows that $T_{1} \subseteq\left(0: S_{1} p_{1}^{n}\right)$. For the reverse inclusion let $s_{1} \in\left(0: S_{1} p_{1}^{n}\right)$. Then there is an element $s_{2} \in S_{2}$ such that $\left(s_{1}, s_{2}\right) \in S$ and $\left(P_{1}^{n} \oplus 0\right)\left(s_{1}, s_{2}\right)=0$. Hence $\left(s_{1}, s_{2}\right) \in T^{\prime}$. Thus $s_{1} \in T_{1}$ and we have equality. Therefore $S_{1}$ is 2-absorbing comultiplication. Similarly, $S_{2}$ is 2-absorbing comultiplication. Conversely, assume that $S_{1}, S_{2}$ are 2-absorbing comultiplication and let $T$ be a 2 -absorbing submodule of $S$. By Lemma 3.1, $T_{1}, T_{2}$ are 2-absorbing submodules of $S_{1}, S_{2}$, respectively.

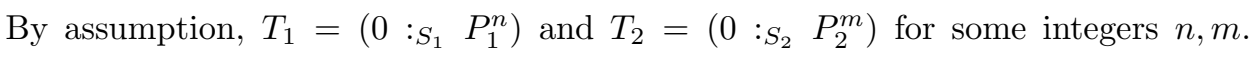
An inspection will show that $T=\left(\begin{array}{lll}0: S & P_{1}^{n} \oplus P_{2}^{m}\end{array}\right)$. Thus $S$ is a 2-absorbing comultiplication $R$-module. 
Lemma 3.5. Let $R$ be the pullback ring as in (1). Then, up to isomorphism, the following separated $R$-modules are indecomposable and 2-absorbing comultiplication:

(i) $S=\left(E\left(R_{1} / P_{1}\right) \rightarrow 0 \leftarrow 0\right),\left(0 \rightarrow 0 \leftarrow E\left(R_{2} / P_{2}\right)\right)$, where $E\left(R_{i} / P_{i}\right)$ is the $R_{i}$-injective hull of $R_{i} / P_{i}$ for $i=1,2$;

(ii) $S=\left(Q\left(R_{1}\right) \rightarrow 0 \leftarrow 0\right),\left(0 \rightarrow 0 \leftarrow Q\left(R_{2}\right)\right)$, where $Q\left(R_{i}\right)$ is the field of fractions of $R_{i}$ for $i=1,2$;

(iii) $S=\left(R_{1} / P_{1}^{n} \rightarrow \bar{R} \leftarrow R_{2} / P_{2}^{m}\right)$ for all positive integers $n, m$.

Proof. By [10, Lemma 2.8], these modules are indecomposable. They are 2absorbing comultiplication by Theorem 2.6 and Theorem 3.4.

Theorem 3.6. Let $R$ be the pullback ring as in (1), and let $S=\left(S_{1} \rightarrow \bar{S} \leftarrow S_{2}\right)$ be an indecomposable separated 2-absorbing comultiplication $R$-module. Then $S$ is isomorphic to one of the modules listed in Lemma 3.5.

Proof. If $\operatorname{abSpec}(S)=\emptyset$, then $\operatorname{abSpec}\left(S_{i}\right)=\emptyset$ by Proposition 3.1 (ii), so $S_{i}=P_{i} S_{i}$ for each $i=1,2$ (see Theorem 2.6, Case 1); hence $S=P S=P_{1} S_{1} \oplus P_{2} S_{2}=S_{1} \oplus S_{2}$. Therefore, $S=S_{1}$ or $S_{2}$ and so $S$ is of type (i) in the list of Lemma 3.5 by Theorem 2.6. So we may assume that $\operatorname{abSpec}(S) \neq \emptyset$. If $S=P S$, then by [10, Lemma 2.7 (i)], $S=S_{1}$ or $S=S_{2}$ and so $S$ is an indecomposable 2-absorbing comultiplication $R_{i}$-module for some $i$, and since $P S=S$, it is of type (ii) by Theorem 2.6. So we may assume that $S \neq P S$.

By Theorem 3.5, $S_{i}$ is a 2-absorbing comultiplication $R_{i}$-module, for each $i=$ 1,2 . Therefore by structure of 2-absorbing comultiplication modules over discrete valuation domain (see Theorem 2.7), $S_{i}=M_{i} \oplus N_{i}$, where $N_{i}$ is a direct sum of copies of $R_{i} / P_{i}^{n}(n \geq 1)$ and $M_{i}$ is a direct sum of copies of $E\left(R_{i} / P_{i}\right)$ and $Q\left(R_{i}\right)$. Then we have $S=\left(N_{1} \rightarrow \bar{S} \leftarrow N_{2}\right) \oplus\left(M_{1} \rightarrow 0 \leftarrow 0\right) \oplus\left(0 \rightarrow 0 \leftarrow M_{2}\right)$. As $S$ is indecomposable and $S \neq P S$, we get that $S=\left(N_{1} \rightarrow \bar{S} \leftarrow N_{2}\right)$. We will see that $S_{i}\left(=N_{i}\right)$ is indecomposable. Then there are positive integers $u, v$ and $w$ such that $P_{1}^{u} S_{1}=0, P_{2}^{v} S_{2}=0$ and $P^{w} S=0$. Choose $s \in S_{1} \cup S_{2}$ with $\bar{s} \neq 0$ and let $o(s)$ denote the least positive integer $k$ such that $P^{k} s=0$ if there is such $k$ and if no such $k o(s)=\infty$ and $o(s)$ minimal among such $s$. Assume $s \in S_{2}$, and so write $s=s_{2}$ and $m=k=o\left(s_{2}\right)$. Now pick $s_{1} \in S_{1}$ with $\bar{s}_{1}=\bar{s}_{2}=\bar{s}$ and $o\left(s_{1}\right)=n$ minimal (so $o\left(s_{2}\right) \neq \infty$ and $o\left(s_{1}\right) \neq \infty$ ). There exists a $s=\left(s_{1}, s_{2}\right)$ such that $o(s)=n_{1}$, $o\left(s_{1}\right)=m_{2}$ and $o\left(s_{2}\right)=k_{1}$. Then $R_{i} s_{i}$ is pure in $S_{i}$ for $i=1,2$ (see [10, Theorem 2.9]). Therefore, $R_{1} s_{1} \cong R_{1} / P_{1}^{m_{2}}$ (resp. $R_{2} s_{2} \cong R_{2} / P_{2}^{k_{1}}$ ) is a direct summand of $S_{1}$ (resp. $S_{2}$ ) since for each $i, R_{i} s_{i}$ is pure-injective. Let $\bar{M}$ be the $\bar{R}$-subspace of $\bar{S}$ generated by $\bar{s}$. Then $\bar{M} \cong \bar{R}$. Let $M=\left(R_{1} s_{1}=M_{1} \rightarrow \bar{M} \leftarrow M_{2}=R_{2} s_{2}\right)$. Then $M$ is an $R$-submodule of $S$ which is 2-absorbing comultiplication by Lemma 
3.5 and is a direct summand of $S$; this implies that $S=M$, and $S$ is as in (iii) in the list of Lemma 3.5 (see [10, Theorem 2.9]).

Corollary 3.7. Let $R$ be the pullback ring as in (1).

(i) Every separated 2-absorbing comultiplication $R$-module $S$ is of the form $S=M \oplus N$, where $M$ is a direct sum of copies of the modules as in (iii), and $N$ is a direct sum of copies of the modules as in (i)-(ii) of Lemma 3.5.

(ii) Every separated 2-absorbing comultiplication R-module is pure-injective.

Proof. Apply Theorem 3.6 and [10, Theorem 2.9].

\section{The non-separated absorbing comultiplication modules}

We continue to use the notation already established, so $R$ is a pullback ring as in (1). In this section we find the indecomposable non-separated 2-absorbing comultiplication modules with finite-dimensional top. It turns out that each can be obtained by amalgamating finitely many separated indecomposable 2-absorbing comultiplication modules. We need the following lemma proved in [17, Proposition 4.1].

Lemma 4.1. Let $R$ be a pullback ring as in (1).

(i) $E(R / P)$ is an indecomposable non-separated 2-absorbing comultiplication $R$-module.

(ii) If $0 \rightarrow K \rightarrow S \rightarrow M \rightarrow 0$ is a separated representation of an $R$-module $M$, then $\operatorname{abSpec}_{R}(S)=\emptyset$ if and only if $\operatorname{abSpec}_{R}(M)=\emptyset$.

Lemma 4.2. Let $R$ be a pullback ring as in (1) and let $0 \rightarrow K \rightarrow S \rightarrow M \rightarrow 0$ be a separated representation of an $R$-module $M$.

(i) If $\left(0:_{R} S\right)=P_{1}^{m} \oplus 0$ for some positive integer $m$, then $M$ is separated.

(ii) If $\left(0:_{R} S\right)=0 \oplus P_{2}^{m}$ for some positive integer $m$, then $M$ is separated.

(iii) (ii) If $\left(0:_{R} S\right)=0$, then $M$ is separated.

Proof. (i) Suppose that $\left(0:_{R} S\right)=P_{1}^{m} \oplus 0$. As $P_{1}^{m} \oplus 0 \subseteq\left(\left(0 \oplus P_{2}\right) S:_{R} S\right)$ and $0 \oplus P_{2} \subseteq\left(\left(0 \oplus P_{2}\right):_{R} S\right)$, we find that $P_{1}^{m} \oplus P_{2} \subseteq\left(\left(0 \oplus P_{2}\right):_{R} S\right)$. It then follows from [14, Proposition 4.3] that $K \subseteq P^{m} S \subseteq\left(P_{1}^{m} \oplus P_{2}\right) S \subseteq\left(0 \oplus P_{2}\right) S$; hence $K=0$ since $K \cap\left(0 \oplus P_{2}\right) S=0$. Thus $M$ is separated. The proof of (ii) is similar.

(iii) An inspection will show that $\left(0:_{R} M\right)=0$. It follows that $\left(P_{1} \oplus 0\right) M \neq 0$ and $\left(0 \oplus P_{2}\right) M \neq 0$. Let $m \in\left(P_{1} \oplus 0\right) M \cap\left(0 \oplus P_{2}\right) M$. If $m=0$, we are done. So suppose that $m \neq 0$. Since $\varphi^{-1}(R m)=\varphi^{-1}(\varphi(R x)=R x$ and $R m \neq 0,0 \rightarrow$ $K \rightarrow R x \rightarrow R m \rightarrow 0$ is a separated representation of $R m$ with $K \subseteq P(R x)$ by [12, 
Lemma 3.1]. By hypothesis, there exist $m_{1}, m_{2} \in M$ and $x \in S$ such that $m=\varphi(x)$ and $m=\left(p_{1}^{s}, 0\right) m_{1}=\left(0, p_{2}^{t}\right) m_{2}$ for some integers $s, t$. Then $\left(p_{1}, 0\right) m=0=\left(0, p_{2}\right) m$ gives $P m=0$, and so $\varphi(P x)=0$; hence $\varphi\left(P_{1} x\right)=\varphi\left(P_{2} x\right)=0$. Since $\varphi$ is oneto-one on $P_{i} S$ for each $i$, we get that $P x=0$; so $K \subseteq P(R x)=0$. Thus $M$ is a separated $R$-module.

Proposition 4.3. Let $R$ be a pullback ring as in (1) and let $M$ be a 2-absorbing comultiplication non-separated $R$-module. Let $0 \rightarrow K \rightarrow S \rightarrow M \rightarrow 0$ be a separated representation of $M$. If $N$ is a non-zero $R$-submodule of $M$, then $M / N$ is a 2absorbing comultiplication R-module.

Proof. Let $L / N$ be a 2-absorbing submodule of $M / N$. Then $L$ is a 2-absorbing submodule of $M$ by Lemma 2.2 (i), so $L=\left(0::_{M} \operatorname{ann}(L)\right)$. Since $\operatorname{ann}(M) \subseteq$ $\operatorname{ann}(L) \neq 0$ and $M$ is a non-separated $R$-module, Lemma 4.2 gives $\operatorname{ann}(L)=$ $P_{1}^{n} \oplus P_{2}^{m}$ for some positive integers $m, n$ (note that if $\operatorname{ann}(M)=0$, then $\operatorname{ann}(S) \subseteq$ $\left.\left(K:_{R} S\right)=\operatorname{ann}(M)=0\right)$. We show that $L / N=\left(0:_{M / N}\left(P_{1}^{n} \oplus P_{2}^{m}\right)\right)$. Let $x+N \in L / N$. Then $\left(P_{1}^{n} \oplus P_{2}^{m}\right) x=0$ gives $\left(P_{1}^{n} \oplus P_{2}^{m}\right)(x+N)=0$; so $x+N \in\left(0:_{M / N}\right.$ $\left.\left(P_{1}^{n} \oplus P_{2}^{m}\right)\right)$. For the reverse inclusion, assume that $y+N \in\left(0:_{M / N}\left(P_{1}^{n} \oplus P_{2}^{m}\right)\right)$. Then $\left(P_{1}^{n} \oplus P_{2}^{m}\right) y \subseteq N \subseteq L$. We claim that $\left(P_{1}^{n} \oplus P_{2}^{m}\right) y=0$. Assume to the contrary, $0 \neq\left(P_{1}^{n} \oplus P_{2}^{m}\right) y \subseteq L$. Then $\left(P_{1}^{2 n} \oplus P_{2}^{2 m}\right) y=0$. Let $s$ be the least positive integer such that $P^{s} y=0$ (so $P^{s-1} y \neq 0$ ). There exists $x \in S$ such that $y=\varphi(x)$ and $\varphi\left(P^{s} x\right)=0$; so $\varphi\left(P_{1}^{s} x\right)=\varphi\left(P_{2}^{s} x\right)=0$. By [26, Proposition 2.3], $\varphi$ is one-to-one on $P_{i} S$ for each $i$, we find that $P_{2}^{s} x=P_{1}^{s} x=0$; hence $P^{s} x=0$. Set $U=P^{s-1} y$. Then $0 \rightarrow K \rightarrow \varphi^{-1}(U)=P^{s-1} x \rightarrow U \rightarrow 0$ is a separated representation of $U$ by [12, Lemma 3.1] such that $K \subseteq P\left(P^{s-1} x\right)=0$ which is a contradiction. Thus $\left(P_{1}^{n} \oplus P_{2}^{m}\right) y=0$, and so we have equality.

Theorem 4.4. Let $R$ be a pullback ring as in (1) and let $M$ be any non-separated $R$-module. Let $0 \rightarrow K \rightarrow S \rightarrow M \rightarrow 0$ be a separated representation of $M$. Then $S$ is 2-absorbing comultiplication if and only if $M$ is 2-absorbing comultiplication.

Proof. By Lemma 4.1 (ii), we may assume that $\operatorname{abSpec}(S) \neq \emptyset$. Suppose that $M$ is a 2-absorbing comultiplication $R$-module and let $T$ be a non-zero 2-absorbing submodule of $S$. Then by [14, Proposition 4.3], $K \subseteq T$, and so $T / K$ is a 2 absorbing submodule of $S / K \cong M$ by Lemma $2.2(\mathrm{i})$. Since $S / K \cong M$ is 2 -absorbing comultiplication, we have $T / K=\left(0:_{S / K} P_{1}^{n} \oplus P_{2}^{m}\right)$ for some integer $m, n$. By an argument like that in [14, Theorem 4.4], we find that $T=\left(0:_{S} P_{1}^{n} \oplus P_{2}^{m}\right)$, and so $S$ is 2-absorbing comultiplication. Conversely, assume that $S$ is a 2-absorbing comultiplication $R$-module. Then $M \cong S / K$ is a 2 -absorbing comultiplication $R$ module by Proposition 4.3. 
Proposition 4.5. Let $R$ be a pullback ring as in (1), and let $M$ be an indecomposable 2-absorbing comultiplication non-separated $R$-module with $M / P M$ finitedimensional top over $\bar{R}$. If $0 \rightarrow K \rightarrow S \rightarrow M \rightarrow 0$ is a separated representation of $M$, then $S$ has finite-dimensional top and is pure-injective.

Proof. Since $S / P S \cong M / P M$ by [10, Proposition 2.6 (i)], we find that $S$ has finite-dimensional top. Now the assertion follows from Theorem 4.4 and Corollary 3.7 .

Let $R$ be a pullback ring as in (1) and let $M$ be an indecomposable 2-absorbing comultiplication non-separated $R$-module with $M / P M$ finite-dimensional over $\bar{R}$. Consider the separated representation $0 \rightarrow K \rightarrow S \rightarrow M \rightarrow 0$. By Proposition 4.3, $S$ is pure-injective. So in the proofs of [10, Lemma 3.1, Proposition 3.2 and Proposition 3.4] (here the pure-injectivity of $M$ implies the pure-injectivity of $S$ by [10, Proposition 2.6 (ii)]) we can replace the statement " $M$ is an indecomposable pure-injective non-separated $R$-module" by " $M$ is an indecomposable 2-absorbing comultiplication non-separated $R$-module": because the main key in those results are the pure-injectivity of $S$, the indecomposability and the non-separability of $M$. So we have the following result:

Corollary 4.6. Let $R$ be a pullback ring as in (1) and let $M$ be an indecomposable 2absorbing comultiplication non-separated $R$-module with $M / P M$ finite-dimensional over $\bar{R}$, and let $0 \rightarrow K \rightarrow S \rightarrow M \rightarrow 0$ be a separated representation of $M$. Then the following hold:

(i) The quotient fields $Q\left(R_{1}\right)$ and $Q\left(R_{2}\right)$ of $R_{1}$ and $R_{2}$ do not occur among the direct summands of $S$.

(ii) $S$ is a direct sum of finitely many indecomposable 2-absorbing comultiplication modules.

(iii) At most two copies of modules of infinite length can occur among the indecomposable summands of $S$.

Before embarking on the proof of the next result let us explain its idea. Let $R$ be a pullback ring as in (1). Let $M$ be any $R$-module and let $0 \rightarrow K \rightarrow S \rightarrow M \rightarrow 0$ be a separated representation of $M$. We have already shown that if $M$ is indecomposable 2-absorbing comultiplication with $M$ finite-dimensional top then $S$ is a direct sum of just finitely many indecomposable separated 2-absorbing comultiplication modules and these are known by Theorem 3.6. In any separated representation $0 \rightarrow K \stackrel{i}{\longrightarrow} S \stackrel{\varphi}{\longrightarrow} M \rightarrow 0$ the kernel of the map $\varphi$ to $M$ is annihilated by $P$, hence 
is contained in the socle of the separated module $S$. Thus $M$ is obtained by amalgamation in the socles of the various direct summands of $S$. This explains Corollary 4.6 (i): the modules $Q\left(R_{1}\right)$ and $Q\left(R_{2}\right)$ have zero socle and so cannot be amalgamated with any other direct summands of $S$ and hence cannot occur in a separated (hence "minimal") representation. So the questions are: does this provide any further condition on the possible direct summands of $S$ ? How can these summands be amalgamated in order to form $M$ ? For the case of finitely generated $R$-modules $M$ these questions are answered by Levy's description [25], see also [26, Section 11]. Levy shows that the indecomposable finitely generated $R$-modules are of two nonoverlapping types which he calls deleted cycle and block cycle types. It is the modules of deleted cycle type which are most relevant to us. Such a module is obtained from a direct summand, $S$, of indecomposable separated modules by amalgamating the direct summands of $S$ in pairs to form a chain but leaving the two ends unamalgamated. Reflecting the fact that the dimension over $\bar{R}$ of the socle of any finitely generated indecomposable separated module is $\leq 2$ each indecomposable summand of $S$ may be amalgamated with at most two other indecomposable summands. Consider the indecomposable separated $R$-modules $S(n, m)=\left(R_{1} / P_{1}^{n} \rightarrow \bar{R} \leftarrow R_{2} / P_{2}^{m}\right)$ with $n, m \geq 2$ (it is generated over $R$ by $\left(1+P_{1}^{n}, 1+P_{2}^{m}\right)$ ). Actually, separated indecomposable $R$-modules also include $R_{1} / P_{1}^{n}$ for $n \geq 2$, which can be regarded up to isomorphism as $S(n, 1)=\left(R_{1} / P_{1}^{n} \rightarrow \bar{R} \leftarrow R_{2} / P_{2}\right)$. Similarly, for $m \geq 2$, $S(1, m)=\left(R_{1} / P_{1} \rightarrow \bar{R} \leftarrow R_{2} / P_{2}^{m}\right)$ is a separated indecomposable $R$-module. Moreover, $R_{1}, R_{2}$ and $R$ themselves can be viewed as separated indecomposable $R$-modules, corresponding to the cases $n=\infty$ and $m=1, n=1$ and $m=\infty$, $n=m=\infty$. Deleted cycle indecomposable $R$-modules are introduced as follows: Let $S$ be a direct sum of finitely many modules $S(i)=S\left(n_{i, 1}, n_{i, 2}\right)$ (with $i<s$ a non-negative integer). Here $n_{i, j} \geq 2$ for every $j<s$ and $j=1$, 2 , with two possible exceptions $i=0, j=1$ and $i=s-1$ and $j=2$, where the values $n_{i, j}=1$ or $\infty$ are allowed. Then amalgamate the direct summands in $S$ by identifying the $P_{2}$-part of the socle of $S(i)$ and the $P_{1}$-part of the socle $S(i+1)$ for every $i<s-1$. For instance, given the separated modules $S_{1}=\left(R_{1} \rightarrow \bar{R} \leftarrow R_{2} / P_{2}^{3}\right)=R a$ with $P_{2}^{3} a=0$ and $S_{2}=\left(R_{1} / P_{1}^{7} \rightarrow \bar{R} \leftarrow R_{2} / P_{2}^{2}\right)=R a^{\prime}$ with $P_{1}^{7} a^{\prime}=0=P_{2}^{2} a^{\prime}$. Then one can form the non-separated module $\left(S_{1} \oplus S_{2}\right) /\left(R\left(p_{2}^{2} a-p_{1}^{6} a^{\prime}\right)=R c+R c^{\prime}\right.$ where $c=a+R\left(p_{2}^{2} a-p_{1}^{6} a^{\prime}\right), c^{\prime}=a^{\prime}+R\left(p_{2}^{2} a-p_{1}^{6} a^{\prime}\right), P_{2}^{3} c=0=P_{1}^{7} c^{\prime}=P_{2}^{2} c$ and $P_{2}^{2} c=P_{1}^{6} c^{\prime}$ which is obtained by identifying the $P_{2}$-part of the socle of $S_{1}$ with the $P_{1}$-part of the socle of $S_{2}$. We will use that same description, but with 2absorbing comultiplication separated modules in place of the finitely generated ones, gives us the non-zero indecomposable 2-absorbing comultiplication non-separated 
$R$-modules. As a consequence, any non-zero indecomposable 2-absorbing comultiplication separated module with 1-dimensional socle may occur only at one of the ends of the amalgamation chain (see [10, Proposition 3.4]). It remains to show that the modules obtained by these amalgamations are, indeed, indecomposable 2-absorbing comultiplication. We do that now and thus complete the classification of the indecomposable 2-absorbing comultiplication non-separated modules with finite-dimensional top.

Theorem 4.7. Let $R=\left(R_{1} \rightarrow \bar{R} \leftarrow R_{2}\right)$ be the pullback of two discrete valuation domains $R_{1}, R_{2}$ with common factor field $\bar{R}$. Then the indecomposable nonseparated 2-absorbing comultiplication modules with finite-dimensional top, up to isomorphism, are the following:

(i) $M=E(R / P)$, the injective hull of $R / P$;

(ii) The indecomposable modules of finite length (apart from $R / P$ which is separated), that is, $M=\sum_{i=1}^{s} R a_{i}$ with

$$
p_{1}^{n_{s}} a_{s}=0=p_{2}^{m_{1}} a_{1}, p_{1}^{n_{i}-1} a_{i}=p_{2}^{m_{i+1}-1} a_{i+1}(1 \leq i \leq s-1)
$$

$m_{i}, n_{i} \geq 2$ except for $m_{1} \geq 1, n_{s} \geq 1$.

(iii) $M=E_{1}+\sum_{i=1}^{s} R a_{i}+E_{2}$ with

$$
a_{0}=p_{2}^{m_{1}-1} a_{1}, b_{0}=p_{1}^{n_{s}-1} a_{s}, p_{1} a_{0}=0=p_{2} b_{0},
$$

and $p_{1}^{n_{i}-1} a_{i}=p_{2}^{m_{i+1}-1} a_{i+1}$ for all $1 \leq i \leq s-1$, where $E_{1} \cong E\left(R a_{0}\right) \cong$ $E\left(R_{1} / P_{1}\right), E_{2} \cong E\left(R b_{0}\right) \cong E\left(R_{2} / P_{2}\right)$ and $m_{i}, n_{i} \geq 2$ except for $m_{1} \geq 1$, $n_{s} \geq 1$.

(iv) $M=E_{1}+\sum_{i=1}^{s} R a_{i}$ with

$$
p_{1}^{n_{s}} a_{s}=0, a_{0}=p_{2}^{m_{1}-1} a_{1}, p_{1} a_{0}=0,
$$

and $p_{1}^{n_{i}-1} a_{i}=p_{2}^{m_{i+1}-1} a_{i+1}$ for all $1 \leq i \leq s-1$, where $E_{1} \cong E\left(R a_{0}\right) \cong$ $E\left(R_{1} / P_{1}\right)$, and $m_{i}, n_{i} \geq 2$ except for $n_{s} \geq 1$,

(v) $M=\sum_{i=1}^{s} R a_{i}+E_{2}$ with

$$
p_{2}^{m_{s}} a_{s}=0, b_{0}=p_{1}^{n_{1}-1} a_{1}, p_{2} b_{0}=0,
$$

and $p_{2}^{m_{i}-1} a_{i}=p_{1}^{n_{i+1}-1} a_{i+1}$ for all $1 \leq i \leq s-1$, where $E_{2} \cong E\left(R b_{0}\right) \cong$ $E\left(R_{2} / P_{2}\right)$, and $m_{i}, n_{i} \geq 2$ except for $m_{s} \geq 1$. 
Proof. Let $M$ be an indecomposable non-separated 2-absorbing comultiplication $R$-module with finite-dimensional top and let $0 \rightarrow K \stackrel{i}{\longrightarrow} S \stackrel{\varphi}{\longrightarrow} M \rightarrow 0$ be a separated representation of $M$. By Corollary 4.6 (iii), $S$ is a direct sum of finitely many indecomposable 2-absorbing comultiplication separated modules. We know already that every indecomposable 2-absorbing comultiplication non-separated module has one of these forms so it remains to show that the modules obtained by these amalgamation are, indeed, indecomposable 2-absorbing comultiplication modules. (i) follows from Lemma 4.1 (i). Since a quotient of any 2-absorbing comultiplication $R$-module is 2 -absorbing comultiplication by Proposition 4.3, they are 2-absorbing comultiplication. The indecomposability follows from $[25,1.9]$ and $[10$, Theorem $3.5]$.

Remark 4.8. $\quad$ (i) Let $R$ be the pullback ring described in Theorem 4.7. Then by [10, Theorem 3.5] and Theorem 4.7, every indecomposable 2-absorbing comultiplication $R$-module with finite-dimensional top is pure-injective.

(ii) This paper includes the classification of indecomposable 2-absorbing comultiplication modules with finite-dimensional top over $k$-algebra $k[x, y: x y=$ $0]_{(x, y)}$.

Acknowledgement. The authors would like to thank the referee for the valuable suggestions and comments.

\section{References}

[1] Y. Al-Shaniafi and P. F. Smith, Comultiplication modules over commutative rings, J. Commut. Algebra, 3(1) (2011), 1-29.

[2] D. M. Arnold and R. C. Laubenbacher, Finitely generated modules over pullback rings, J. Algebra, 184(1) (1996), 304-332.

[3] I. Assem, D. Simson and A. Skowroński, Elements of the Representation Theory of Associative Algebras, Vol. 1, Techniques of Representation Theory, London Math. Soc. Student Texts, 65, Cambridge University Press, Cambridge, 2006.

[4] A. Badawi, On 2-absorbing ideals of commutative rings, Bull. Austral. Math. Soc., 75(3) (2007), 417-429.

[5] A. Badawi, U. Tekir and E. Yetkin, On 2-absorbing primary ideals in commutative rings, Bull. Korean Math. Soc., 51(4) (2014), 1163-1173.

[6] A. Badawi, U. Tekir and E. Yetkin, On weakly 2-absorbing primary ideals of commutative rings, J. Korean Math. Soc., 52(1) (2015), 97-111.

[7] H. Bass, On the ubiquity of Gorenstein rings, Math. Z., 82 (1963), 8-28. 
[8] M. C. R. Butler and C. M. Ringel, Auslander-Reiten sequences with few middle terms, with applications to string algebras, Comm. Algebra, 15(1-2) (1987), 145-179.

[9] Ju. A. Drozd, Matrix problems and categories of matrices, in Russian, Investigations on the theory of representations, Zap. Naučn. Sem. Leningrad. Otdel. Mat. Inst. Steklov. (LOMI), 28 (1972), 144-153.

[10] S. Ebrahimi Atani, On pure-injective modules over pullback rings, Comm. Algebra, 28(9) (2000), 4037-4069.

[11] S. Ebrahimi Atani, On secondary modules over Dedekind domains, Southeast Asian Bull. Math, 25(1) (2001), 1-6.

[12] S. Ebrahimi Atani, On secondary modules over pullback rings, Comm. Algebra, 30(6) (2002), 2675-2685.

[13] S. Ebrahimi Atani, Indecomposable weak multiplication modules over Dedekind domains, Demonstratio Math., 41(1) (2008), 33-43.

[14] R. Ebrahimi Atani and S. Ebrahimi Atani, Comultiplication modules over a pullback of Dedekind domains, Czechoslovak Math. J., 59 (2009), 1103-1114.

[15] R. Ebrahimi Atani and S. Ebrahimi Atani, Weak comultiplication modules over a pullback of commutative local Dedekind domains, Algebra Discrete Math., 1 (2009), 1-13.

[16] S. Ebrahimi Atani and F. Farzalipour, Weak multiplication modules over a pullback of Dedekind domains, Colloq. Math., 114(1) (2009), 99-112.

[17] S. Ebrahimi Atani and M. Sedghi Shanbeh Bazari, Absorbing multiplication modules over pullback rings, Int. Electron. J. Algebra, 21 (2017), 76-90.

[18] A. Facchini, Fiber products and Morita duality for commutative rings, Rend. Sem. Mat. Univ. Padova, 67 (1982), 143-159.

[19] A. Facchini and P. Vámos, Injective modules over pullbacks, J. London Math. Soc., 31 (1985), 425-438.

[20] J. Haefner and L. Klingler, Special quasi-triads and integral group rings of finite representation type I, J. Algebra, 158(2) (1993), 279-322.

[21] J. Haefner and L. Klingler, Special quasi-triads and integral group rings of finite representation type II, J. Algebra, 158(2) (1993), 323-374.

[22] I. Kaplansky, Modules over Dedekind rings and valuation rings, Trans. Amer. Math., Soc., 72 (1952), 327-340.

[23] L. Klingler, Integral representations of groups of square-free order, J. Algebra, 129(1) (1990), 26-74.

[24] L. S. Levy, Modules over pullbacks and subdirect sums, J. Algebra, 71(1) (1981), $50-61$. 
[25] L. S. Levy, Mixed modules over ZG, G cyclic of prime order, and over related Dedekind pullbacks, J. Algebra, 71(1) (1981), 62-114.

[26] L. S. Levy, Modules over Dedekind-like rings, J. Algebra, 93(1) (1985), 1-116.

[27] R. L. McCasland, M. E. Moore and P. F. Smith, On the spectrum of a module over a commutative ring, Comm. Algebra, 25(1) (1997), 79-103.

[28] M. E. Moore and S. J. Smith, Prime and radical submodules of modules over commutative rings, Comm. Algebra, 30(10) (2002), 5037-5064.

[29] L. A. Nazarova and A. V. Roiter, Finitely generated modules over a dyad of two local Dedekind rings, and finite groups which possess an abelian normal divisor of index p, Izv. Akad. Nauk SSSR Ser. Mat., 33 (1969), 65-89.

[30] Sh. Payrovi and S. Babaei, On the 2-absorbing submodules, Iran. J. Math. Sci. Inform., 10(1) (2015), 131-137.

[31] M. Prest, Model Theory and Modules, London Mathematical Society Lecture Note Series, 130, Cambridge University Press, Cambridge, 1988.

[32] M. Prest, Ziegler spectra of tame hereditary algebras, J. Algebra, 207(1) (1998), 146-164.

[33] C. M. Ringel, Some algebraically compact modules I, Abelian groups and modules (Padova, 1994), Math. Appl., 343, Kluwer Acad. Publ., Dordrecht, (1995), 419-439.

[34] C. M. Ringel, The Ziegler spectrum of a tame hereditary algebra, Colloq. Math., 76(1) (1998), 105-115.

[35] D. Simson, Linear Representations of Partially Ordered Sets and Vector Space Categories, Algebra, Logic and Applications, Vol. 4, Gordon and Breach Science Publishers, Montreux, 1992.

[36] D. Simson, On Corner type endo-wild algebras, J. Pure Appl. Algebra, 202(1-3) (2005), 118-132.

[37] R. B. Warfield, Jr., Purity and algebraic compactness for modules, Pacific J. Math., 28 (1969), 699-719.

[38] A. N. Wiseman, Projective modules over pullback rings, Math. Proc. Cambridge Philos. Soc., 97(3) (1985), 399-406.

[39] A. Yousefian Darani and F. Soheilnia, 2-Absorbing and weakly 2-absorbing submodules, Thai J. Math., 9(3) (2011), 577-584.

[40] M. Ziegler, Model theory of modules, Ann. Pure Appl. Logic, 26(2) (1984), 149-213. 
Shahabaddin Ebrahimi Atani (Corresponding Author), Saboura Dolati Pish Hesari,

Mehdi Khoramdel and Maryam Sedghi Shanbeh Bazari

Department of Mathematics

Faculty of Science

University of Guilan

P.O. Box 1914, Rasht, Iran

e-mails: ebrahimi@guilan.ac.ir (S. Ebrahimi Atani)

saboura_dolati@yahoo.com (S. Dolati Pish Hesari)

mehdikhoramdel@gmail.com (M. Khoramdel)

ms.maryamsedghi55@gmail.com (M. Sedghi Shanbeh Bazari) 